\title{
Human-robot collaborative assembly in cyber-physical production: classification framework and implementation
}

\author{
Xi Vincent Wang ${ }^{\mathrm{a}, *}$, Zsolt Kemény ${ }^{\mathrm{b}}$, József Váncza $(1)^{\mathrm{b}, \mathrm{c}}$, Lihui Wang $(1)^{\mathrm{a}}$ \\ a Department of Production Engineering, KTH Royal Institute of Technology, Sweden \\ ${ }^{\mathrm{b}}$ Institute for Computer Science and Control, Hungarian Academy of Sciences, Hungary \\ ${ }^{c}$ Dept. of Manufacturing Science and Engineering, Budapest University of Technology and Economics, Budapest, Hungary
}

The production industry is moving towards the next generation of assembly, which is conducted based on safe and reliable robots working in the same workplace alongside with humans. Focusing on assembly tasks, this paper presents a review of human-robot collaboration research and its classification works. Aside from defining key terms and relations, the paper also proposes means of describing human-robot collaboration that can be relied on during detailed elaboration of solutions. A human-robot collaborative assembly system is developed with a novel and comprehensive structure, and a case study is presented to validate the proposed framework.

Assembly, man-machine system, human-robot collaboration

\section{Introduction}

Industrial production is nowadays experiencing changes that shift the emphasis of production-and related R\&D worktowards increasing flexibility and responsiveness of production processes, facilities and entire production networks. Among the drivers of these changes, the key objectives are decreasing desired lead time and growing customisation, leading to higher diversity and more frequent changes of products, components and tasks to be handled within the same production unit.

These trends are expected to affect the way both humans and machines are put to work-most importantly, the meaningful combination of human and robot skills is beginning to gain emphasis. The latter development aligns well with the shift towards more local autonomy in production processes: while certain routine tasks or specific skills can be effeciently supported by automation, local decisions or exceptional intervention often require a "human touch" due to the extraordinary characteristics of the given situation, the complexity, or the implicit nature of knowledge to be relied on in finding a viable solution in a limited time, with bounded resources at hand.

The combination of human and artificial resources has not been part of mainstream automation practice where (1) robots and humans are generally kept away from each other, and (2) humans must adhere to work procedures as rigid as the rest of the automated production environment. Symbiotic Human-Robot Collaboration (HRC) steps beyond these limitations but requires a more responsive, transparent and accessible environment backed by more computational intelligence [1]-[3].

Information and Communication Technology (ICT) has significantly changed assembly systems in the past years, partly due to the massive connectivity of components and actors (LAN, Wi-Fi, Bluetooth, near field communication, etc.), and partly due to increasing process observability and local computing capacity in smart devices (automatic identification, sensors, wearable devices, smart tags, etc.). The close and multi-directional interaction of virtual and physical entities forms a cyber-physical system where automated components and humans can be integrated in a cybernetic and collaborative environment combining their complementary strengths instead of mutual restriction of their potentials [4].
Robots exhibit high precision and repeatability, can handle heavy loads and operate without performance deterioration even in difficult or dangerous environments. However, robot control systems quickly reach their limits in recognizing and handling unexpected situations, as reflected by the relatively rigid plans and robot programs widespread in today's automated systems.

Humans tackle unexpected situations better, are aware of a much larger part of the environment than formally declared, and show more dexterity in complex or sensitive tasks. Humans, however, are more prone to error, stress or fatigue [5], and their employment underlies strict health and safety regulations.

With technologies able to bridge the gaps in skills and operational characteristics, it is now becoming possible to rely on robots as collaborating partners instead of-potentially hazardous-tools [6]. The appearance of off-the-shelf industrial robots certified for operating alongside humans is a sign of HRC gaining acceptance and spreading in industrial production.

Meanwhile the theoretical and technological supports for HRC are still undergoing notable development. A systematic approach to solutions involving HRC requires an efficient framework, and methodologies for elaborating feasible solutions. Contributing to these, the paper proposes a structured classification and solution framework, illustrated by practical examples.

\section{State of the art in HRC and its classification}

HRC research has been ongoing for decades, with service robots and vehicles operating in unstructured environment being the subject of most intense interest. Industrial production has been a minority field in this regard, partly due to health and safety regulations limiting HRC in practice. Yet, recent results indicate both the revision of established views and regulations, and intensified research of HRC in production scenarios.

\subsection{Human-robot collaboration for assembly}

Industrial robots had been expected to work as the assistant of human workers for a long time, comprising a fast and automatic assembly system and collaborative manufacturing environment [7]. Different robot and gripper structures are developed to assist workers on the assembly line. In general, most of the tasks 

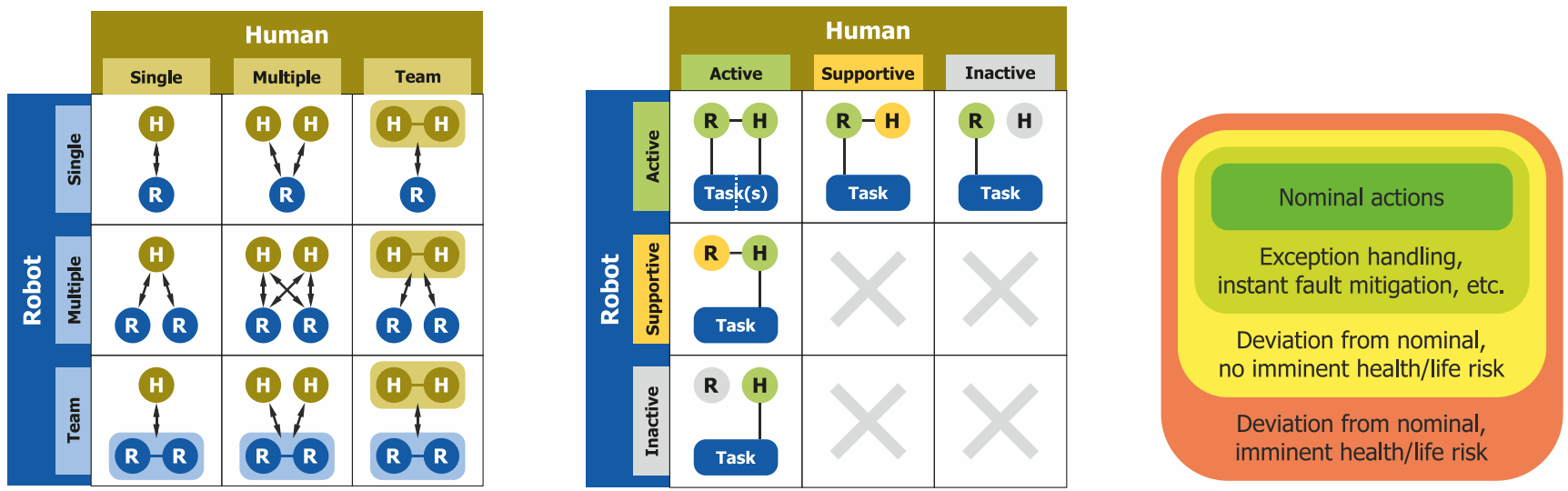

Fig. 1. Classification schemes of human-robot collaboration with regard to agent multiplicity (left), initiative (middle), and alignment of human actions with the nominal process definition (right).

focused on holding an object for the person, laying it aside or retrieving it on demand [7],[8]. In recent years, the production engineering society also gave considerable attention to the collaborative systems in assembly lines. Human-machine and human-robot interactions have been identified as a feasible solution especially suitable for heavy and bulk component handling [9]. Morioka and Sakakibara [2] proposed an assembly system based on HRC. The cooperative parts feeding station is established based on information support and safety management mechanisms. At the control level, Krüger et al. [10] proposed a framework design for stable and robust interaction control. Intuitive programming mechanisms were developed for both online and offline programming via gestures and voices [11]. For the satisfaction of human needs, augmented reality was also deployed in the factory for virtual assembly, assembly guidance, training, maintenance, etc. [12].

\subsection{Classification of human-robot collaboration}

Answering the need for a systematic analysis of HRC requirements and adequate solutions, several considerations have appeared to classify or characterise individual cases [8]. Due to its specific constraints, industrial production usually occupies a mere subset of possibilities. While some characteristics are quantitative, most of the studies highlighted a small number of properties that define distinct classes of HRC instances:

- Temporal and spatial relation of collaborating humans and robots (agents in a more generic sense)-while this shows wide variation in cases like teleoperation or assisted vehicle steering [13], it is assumed in industrial production that the agents (partially) share the same space, even though their activity over time does not necessarily overlap [7]. Close collaboration with physical contact [14]-e.g., common handling of large workpieces-does, naturally, require colocation and simultaneous operation [15].

- Agent multiplicity can be covered to its full diversity by industrial HRC applications. Literature commonly distinguishes between single, multiple, and team (Fig. 1 left), the latter being a group acting by consensus or coordination, and interacting with the environment and other agents in a specified way (e.g., via a "spokesman"). Multiple agents can compete for resources and other agents' services (e.g., one robot serving several manned workstations).

- Agent autonomy and closely related leader-follower relationships express how much of robot action is directly determined by human agents, or which agent takes the lead in the given task. Partitioning along autonomy or initiative can vary depending on the application field [16]. In an industrial context, inactive (resting), active (leading), and supportive (following) behaviour can be distinguished, and many of the current considerations assume that these roles are assigned before task execution (Fig. 1 middle). In some cases, adaptive agents are also contemplated that assign leader/follower roles on-the-fly-these have gained little practical significance so far, and will be omitted in this paper.

Other aspects, such as modes of sensing, interaction, (mutual) awareness are typically treated as independent (orthogonal) characteristics that rarely form distinct classes.

\section{Requirements of symbiotic HRC in assembly}

\subsection{Symbiotic HRC structure in assembly}

Symbiotic collaboration is set aside from conventional HRC by several key characteristics:

- intuitive and multimodal programming environment: workers do not need prior in-depth knowledge of the system,

- zero-programming: ideally, the workers can work with the robots via gestures, voice commands, and other forms of natural inputs without the need of coding,

- immersive collaboration: with the help of different devices, e.g. screens, goggles, wearable displays, the workers can collaborate with the robots with actively engaged senses, and

- context/situation dependency: the system should be capable of interleaving autonomous human with robot decisions based on trustworthy inputs from on-site sensors and monitors inspecting both humans and robots.

The systematic elaboration of solutions for such collaborative cases requires an analysis and synthesis framework containing (1) means to classify and characterise the problem, and (2) solution templates and guidelines for elaboration of a solution seamlessly fitting into existing production premises.

Fundamental elements in characterising an HRC scenario are:

- The actors, i.e., robots and humans either actively taking part in the production process, or occupying a part of the available space, along with multiplicities and roles that outline where prioritising or conflict resolution is needed. Agent characteristics also include communication and sensing modes, geometry, kinematic and dynamic properties, timing characteristics, and, especially in the case of humans, layers of possible actions (nominal, corrective, deviating; Fig. 1 right). Note that actions causing imminent health/life risk are usually caught by dedicated safety functionalities that are often independent of the rest of the HRC support system and can instantly override it-in this case, such actions are not dealt with in the context of HRC. 


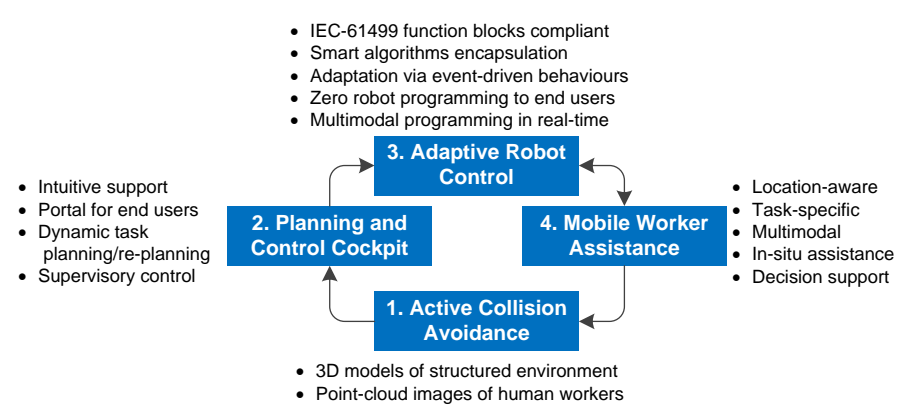

Fig. 2. A symbiotic approach for HRC-based assembly/packaging system

- The work environment includes resources that are needed for production but do not take an active role, as well as artefacts (e.g., stationary structures) and conditions (ambient light, noise, etc.) affecting viable solutions.

- The workpieces and operations are often in inseparable interdependence. Both can have variations to be handled during production (e.g., alternative components or subassemblies). Even an unambiguous set of components can have multiple orders of assembly [17], and the particular choice may depend on actors and resources at hand.

A systematic approach to constructing a symbiotic HRC environment can be understood as a series of analysis and synthesis steps starting with a formal description of tasks, agents and premises, proceeding towards a feasible solution in a partly automated way, while providing the possibility of reiteration and introduction of implicit knowledge at points of manual intervention into the elaboration process. While such mixedinitiative approaches are found in fully automated cases too [18][19], introducing humans in a collaborative context makes the procedure more demanding due to the diversity of actions arising from increased human autonomy. The result of the process is a production environment whose components and structure enable efficient symbiotic HRC.

The symbiotic system structure for HRC-based assembly/ packaging is suggested in [20]. As shown in Fig. 2, the closed-loop architecture consists of Active Collision Avoidance, Planning and Control Cockpit, Adaptive Robot Control, and Mobile Worker Assistance modules, while related supporting technologies are also identified in the figure. The collision avoidance module combines sensor-driven virtual 3D models with real images from vision sensors for efficient perception. Next, the structured robotic environment is established in 3D for the dynamic task planning and re-planning of human-robot shared tasks in a shared workplace. IEC-61499-based function blocks are utilised for adaptive robot control. As the output, the function blocks are automatically converted into robot control codes. Additionally, the workers are supported by in-situ assistance devices-e.g. 3D goggles, headsets, adaptive interfaces-for intuitive and ergonomic interaction.

\subsection{Services to support symbiotic HRC}

Due to the growing reliance on improved process transparency and the evolving capabilities of ICT resources, the virtual representation of manufacturing processes is undergoing rapid development [4]. More and more often, a complete virtual model of an entire production unit is created for surveillance, evaluation, planning and manipulation of the production environment-this is referred to as a digital twin of the physical system. This form of representation can be connected to the physical world in a bidirectional manner, i.e., sensors allow the digital twin to closely follow physical changes, and actuators coupled to the virtual representation enable the physical environment to copy the actions taken in the virtual counterpart.

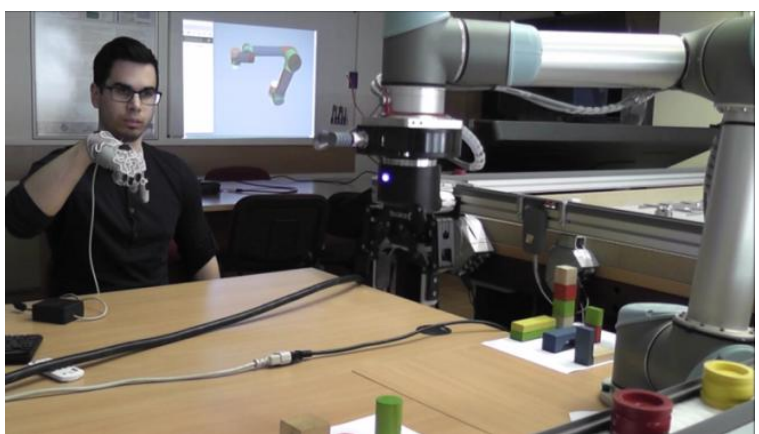

Fig. 3. Human-robot collaborative environment with gesture control interface (left), presentation of a digital twin (centre background), and a physical robot (right).

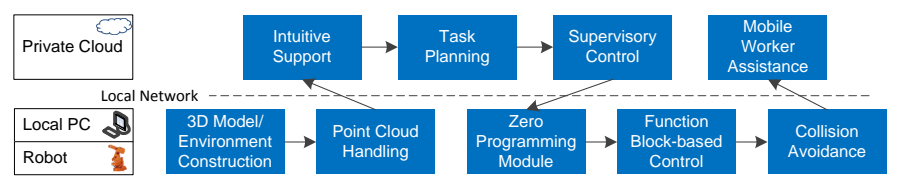

Fig. 4. Task assignment between local host and private cloud

The digital twin can be coupled with additional computational intelligence to handle the diversity of autonomous human actions, or provide cognitive support-e.g., real-time scene assessment, revealing hidden aspects via visualisation or other humaninterpretable feedback-assisting humans in their participation in the production process [21][22]. Fig. 3 shows the result of advanced interfaces and a digital twin in action.

Another important potential of a virtual representation coupled with computational intelligence is that concrete process execution instances on the shop-floor level are no longer detached from long-term engineering activities or preliminary training of workforce [23]. In fact, the virtual representation gradually enriched during the engineering procedure becomes part of the virtual subsystem supporting the production process, enabling the bidirectional transfer of implicit or tacit knowledge, continuous assessment and improvement of processes, and engineering support for process changes by re-planning, offline programming, optimisation and simulation prior to live implementation of modified processes.

\subsection{Cyber-physical systems and Cloud}

Based on the collaborative technologies and services mentioned above, the shared workspace between humans and robots can be considered an advanced cyber-physical system, which is supported by the dynamic control algorithms and online monitoring devices. The physical and software components are tightly coupled to each other while the physical world (robots, monitors, products, etc.) is reflected and controlled by the cyber world (3D models, monitoring data, software, task plans, and so forth). In practice, the interactions between the cyber and physical worlds can be further improved with the help of the latest ICT achievements, e.g., cloud. The cloud technology provides flexible and elastic computing services for multiple types of functions. Connected to the physical devices, it offers broader connectivity and data storage/management resources. Meanwhile, the cyber function mentioned above, e.g. data processing, simulation, and optimisation, can also be executed in the cloud by its stronger computing power. Thus it supports the HRC system as a whole in the backend. To maximise the system performance, the real-time control tasks can be deployed in the local controllers, while the resource-insensitive tasks, like task planning, supervisory control, and mobile worker assistance, can be migrated to the remote computing clouds (Fig. 4). The task balancing between the cloud and local computing units is decided according to a task score mechanism. The latter is obtained from 


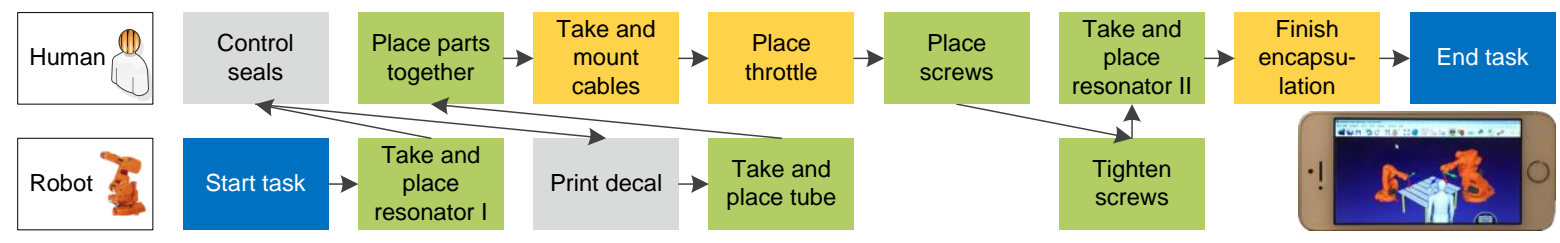

Fig. 5. An engine assembly case based on the HRC classification, powered by the cloud in the backend with mobile access

the score of several criteria, for example, whether the assigned task is time-critical, computing resource-demanding, or in real time. The eligibility is defined based on the nature of a robotic task, along with the criterion weight. Thus based on the result of the total score, each step within an assembly task is distributed to the local host and private cloud.

\section{Implementation and case study}

To validate and evaluate the symbiotic HRC system discussed above, the proposed system is developed towards three industrial scenarios i.e. food packaging, aeronautic component assembly, and automotive engine assembly. Additionally, a private cloud structure is established to provide the computing and access capability, while the knowledge and data is maintained within the fences of a company. In this way, the intellectual property is secured, and the users within the enterprise are able to access and interact with the system without the necessity of installing extra applications on their local computers.

A simplified assembly task is illustrated in Fig. 5 for the supercharger assembly as a part of the automotive engine. The steps within the task are classified based on the schemes developed above. Some of the steps are active HRC actions, e.g. place and hold parts and tighten screws, while some are supportive for the preparation of the next step, e.g., take and place. The wide availability of the HRC system also includes access to general mobile devices, e.g., smartphones and tablets (Fig. 5 bottom right), while the planning and worker assistance are powered by the private cloud in the backend. The assembly plans are validated by geometric reasoning [17]. At this stage of implementation, the main contribution is to validate the feasibility of transforming conventional industrial robotic cells into collaborative environments based on the symbiotic system architecture discussed in section 3. Compared with the investment on new devices which are designed as collaborative robots, the legacy industrial robots have higher speed, bigger payload and better stiffness. In the near future, the engine assembly case will be further evaluated in multiple scales, including cycle time, assembly quality, ergonomic analysis, operator feedback and stress evaluation.

\section{Discussions and conclusions}

Recent trends in assembly point towards an adaptive, flexible and integrated environment where humans and machines share the same workspace. Symbiotic human-robot collaboration enables humans and robots to combine their complementary strengths instead of limiting each other. Such a collaborative environment can be set up with a systematic analysis and synthesis approach in a structured procedure by classifying the collaboration case, determining the requirements, and combining infrastructural elements to a functional solution. The paper presented a possible way of characterising a case, summarised typical requirements, and highlighted which sensing, computational, control and communication resources can be combined to a solution of suggested structure. This paper showed an example of a symbiotic collaborative environment deploying solutions and resources that have a growing impact on production systems, most importantly, novel user interfaces enabling immersive presence and intuitive operation, a comprehensive virtual representation of the production environment and its actors (digital twin), and multiple computational resources that allow local execution with real-time guarantees or assignment to distributed (cloud) services.

\section{Acknowledgement}

Research presented in the paper is supported by the EU H2020 SYMBIO-TIC project of grant No. 637107.

\section{References}

[1] Bicchi A, Peshkin MA, Colgate JE (2008) Safety for physical human-robot interaction. Springer Handbook of Robotics, 1335-1348, Springer.

[2] Morioka M, Sakakibara S (2010) A new cell production assembly system with human-robot cooperation. CIRP Annals-Manufacturing Technology 59(1):912

[3] Wilcox R, Nikolaidis S, Shah J (2012) Optimization of temporal dynamics for adaptive human-robot interaction in assembly manufacturing. Proc. of Robotics Science and Systems VIII, 441-448.

[4] Monostori L, Kádár B, Bauernhansl T, Kondoh S, Kumara S, Reinhart G, Sauer O, Schuh G, Sihn W, Ueda K (2016) Cyber-physical systems in manufacturing. CIRP Annals-Manufacturing Technology 65(2):621-641.

[5] Arai T, Kato R, Fujita M (2010) Assessment of operator stress induced by robot collaboration in assembly. CIRP Annals-Manufacturing Technology 59(1):5-8.

[6] Fong T, Thorpe C, Baur C (2003) Collaboration, dialogue, human-robot interaction. in Robotics Research, 255-266, Springer.

[7] Krüger J, Lien TK, Verl A (2009) Cooperation of human and machines in assembly lines. CIRP Annals-Manufacturing Technology 58(2):628-646.

[8] Yanco HA, Drury J (2004) Classifying human-robot interaction: an updated taxonomy. IEEE International Conference on Systems, Man and Cybernetics 3:2841-2846.

[9] Michalos G, Makris S, Papakostas N, Mourtzis D, Chryssolouris G (2010) Automotive assembly technologies review: challenges and outlook for a flexible and adaptive approach. CIRP Journal of Manufacturing Science and Technology 2(2):81-91.

[10] Krüger J, Schreck G, Surdilovic D (2011) Dual arm robot for flexible and cooperative assembly. CIRP Annals-Manufacturing Technology 60(1):5-8.

[11] Michalos G, Makris S, Spiliotopoulos J, Misios I, Tsarouchi P, Chryssolouris G (2014) ROBO-PARTNER: Seamless Human-Robot cooperation for intelligent, flexible and safe operations in the assembly factories of the future. Procedia CIRP 23:71-76.

[12] Nee A, Ong S, Chryssolouris G, Mourtzis D (2012) Augmented reality applications in design and manufacturing. CIRP Annals-Manufacturing Technology 61(2):657-679.

[13] Burke JL, Murphy RR, Rogers E, Lumelsky VJ, Scholtz J (2004) Final report for the DARPA/NSF interdisciplinary study on human-robot interaction. IEEE Trans. on Systems, Man, and Cybernetics, Part C 34(2):103-112.

[14] De Santis A, Siciliano B, De Luca A, Bicchi A (2008) An atlas of physical humanrobot interaction. Mechanism and Machine Theory 43(3):253-270.

[15] Pellegrinelli S, Moro F L, Pedrocchi N, Tosatti L M, Tolio T (2016) A probabilistic approach to workspace sharing for human-robot cooperation in assembly tasks. CIRP Annals-Manufacturing Technology 65(1):57-60.

[16] Bruemmer DJ, Few DA, Boring RL, Marble JL, Walton MC, Nielsen CW (2005) Shared understanding for collaborative control. IEEE Trans. on Systems, Man, and Cybernetics-Part A: Systems and Humans 35(4):494-504.

[17] Kardos C, Kovács A, Váncza J (2016) Towards feature-based human-robot assembly process planning. Procedia CIRP 57:516-521.

[18] Erdős G, Kardos Cs, Kemény Zs, Kovács A, Váncza J (2016) Process planning and programming for robotic remote laser welding systems. International Journal of Computer Integrated Manufacturing 29(12):1287-1306.

[19] Ceglarek D, Colledani M, Váncza J, Kim DY, Marine C, Kogel-Hollacher M, Mistry A, Bolognese L (2015) Rapid deployment of remote laser welding processes in automotive assembly systems. CIRP Annals-Manufacturing Technology 64(1):389-394.

[20] SYMBIO-TIC Project (2015) EU SYMBIO-TIC Project-Symbiotic HumanRobot Collaborative Assembly: Technologies, Innovations and Competitiveness.

[21] Ong SK, Wang ZB (2011) Augmented assembly technologies based on 3D barehand interaction. CIRP Annals-Manufacturing Technology 60(1):1-4.

[22] Makris S, Karagiannis P, Koukas S, Matthaiakis A S (2016), Augmented reality system for operator support in human-robot collaborative assembly. CIRP Annals-Manufacturing Technology 65(1):61-64.

[23] Mikusz M (2014) Towards an understanding of cyber-physical systems as industrial software-product-service systems. Procedia CIRP 16:385-389. 